УДК 007

\title{
ОСНОВНЫЕ ЭТАПЫ МОНИТОРИНГА ПО ПОИСКУ \\ И ОБНАРУЖЕНИЮ СКРЫТЫХ АТАК \\ В ИНФОРМАЦИОННЫХ СИСТЕМАХ
}

Коцарева Инесса Сергеевна

Рябова Елисавета Вадимовна

студенты

Симонян Арсен Рафикович

к.ф-м.н., доцент

Научный руководитель: Симаворян Симон Жоржевич

к.Т.н., доцент

ФБГОУ ВО «Сочинский государственный университет»

Аннотация: Статья посвящена разработке основных этапов мониторинга по поиску и обнаружению скрытых атак в информационных системах (ИС) различного назначения. Проблема поиска/обнаружения скрытых атак/вторжений в ИС постоянно актуальна в том плане, что злоумышленник регулярно пытается обойти систему защиты, используя новые технологии скрытого обхода системы информационной безопасности, на основе ранее не известных средств и методов вторжения, которые раннее не были зарегистрированы в известных базах сигнатур атаки. Рассмотрены возможности использования таких классов методов как: статистический анализ, искусственный интеллект, нейронные сети, эвристический анализ. Таким образом, предметом исследования является задача по эффективному поиску и обнаружению скрытых атак в ИС. Объектом исследования являются системы информационной безопасности ИС.

Методологические исследования по разработке этапов противодействия скрытым атакам и выявлению особенностей их противодействия базируются на методах статистического анализа, искусственного интеллекта, нейронных сетей и эвристического анализа информационной безопасности. Основным результатом, полученным в работе, является сформированный перечень этапов по организации процесса мониторинга скрытых атак, которые можно разбить на функционально независимые и содержательно разные базовые этапы, реализуемые в разные промежутки времени функционирования 
системы информационной безопасности ИС. Сделан вывод о том, что из рассмотренных классов методов наиболее эффективными являются методы искусственного интеллекта и нейронные сети.

Ключевые слова: защита информации, скрытая атака, система обнаружения вторжений, информационная безопасность, искусственный интеллект, нейронные сети, мониторинг информационной безопасности, системный подход, информационная система, методы искусственного интеллекта.

\title{
MAIN STAGES OF MONITORING FOR SEARCHING AND DETECTING OF HIDDEN ATTACKS IN INFORMATION SYSTEMS
}

\section{Kotcareva Inessa Sergeevna Ryabova Elisaveta Vadimovna Simonyan Arsen Rafikovich Simavoryan Simon Zhorzhevich}

\begin{abstract}
The article is devoted to the development of the main stages of monitoring for searching and detecting of hidden attacks in information systems (IS) of various destinations. The problem of searching / detecting hidden attacks / intrusions in IS is constantly relevant in the sense that a malicious person regularly tries to bypass the protection system using new technologies of hidden bypassing the information security system, based on previously unknown means and methods of intrusion that were not registered in known databases of signatures of attack. There are considered the possibilities of using such classes of methods as: statistical analysis, artificial intelligence, neural networks, heuristic analysis. Thus, the subject of this research is the problem of efficient search and detection of hidden attacks in IS. The object of the research is the information security systems of IS.

Methodological researches on the development of stages of countering hidden attacks and identifying the features of their countermeasures are based on methods of statistical analysis, artificial intelligence, neural networks and heuristic analysis of information security. The main result obtained in the work is the formed list of stages for organizing the monitoring process of hidden attacks, which can be divided into functionally independent and substantively different basic stages, that are implemented at different intervals of time of information security system of IS
\end{abstract}


functioning. It is concluded that among the considered classes of methods, the most effective are artificial intelligence methods and neural networks.

Key words: information protection, hidden attack, intrusion detection system, information security, artificial intelligence, neural networks, information security monitoring, systemic approach, information system, artificial intelligence methods.

\section{Введение}

Основным процессом по информационной безопасности (ИБ), отвечающим за поиск/обнаружение скрытых атак и вторжений, является процесс мониторинга систем обработки данных и информационной безопасности [1, с. 71]. Процесс противодействия любым угрозам разбивается на два этапа: 1) поиск/обнаружение атаки и 2) нейтрализация атаки, т.е. локализация атаки и ликвидация последствий атаки. По своей сути эти этапы слабо зависимы, поскольку методы обнаружения атак, и методы их нейтрализации совершенно различны. Как следствие, решение задачи обнаружения атаки, вообще говоря, не гарантирует ее нейтрализацию. Более того, нейтрализация атаки может произойти и без ее обнаружения, например, в результате проведения плановых профилактических процедур. И, поскольку, в подавляющем большинстве случаев противодействие атаке оказывается возможным только после её обнаружения, то вопрос системного противодействия скрытым атакам в информационных системах остаётся открытым, а его решение востребованным и актуальным. Отметим, что современные средства защиты информации обычно содержат как процедуры обнаружения, так и их нейтрализации, примером являются антивирусные программные средства. Заметим, что мониторинг - основной процесс по информационной безопасности, отвечающий за поиск/обнаружение скрытых атак. Мониторинг может осуществляться как с помощью ранее известных баз сигнатур атаки, так и с помощью методов поиска ранее не известных атак. В данной работе нас интересует именно задача поиска/обнаружения скрытых атак с помощью методов поиска ранее не известных атак по требованиям ИБ.

\section{Основная часть}

Непосредственно процесс поиска/мониторинга скрытых атак можно разбить на следующие функционально независимые и содержательно разные базовые этапы, реализуемые в разные промежутки времени функционирования ИС: 
Этап 1. Сбор текущих динамических данных и получение аналитических данных по выявлению скрытых атак. То есть получение данных в результате проведения анализа накопленных имеющихся данных и других стационарных данных в момент мониторинга информационной системы. Данные должны быть качественными по характеристикам, относящимся к их: а) полноте, б) достоверности, в) актуальности, г) надежности. Отметим, что эти данные должны охватывать не только ИС, но все ее окружение, которое может как-то воздействовать на систему обработки данных. Фиксироваться должны любые сколь угодно незначительные отклонения, изменения и особенности в процессе функционирования ИС и ее окружения, включая систему информационной безопасности. Основной итог реализации данного этапа: максимально полный сбор информации об ИС и ее окружении.

Этап 2. Проведение глубокого и полномасштабного анализа полученной информации по всем существующим методам. Поскольку нет универсальных механизмов эффективного обнаружения скрытых угроз, то используются все возможные на данный момент методы выявления атак в предположении, что хотя бы один из этих методов позволит выявить скрытую атаку. Если хотя бы один из методов обнаруживает определенные отклонения или несоответствия типовой ситуации в системе, то немедленно проводится дополнительный детальный сбор данных по всем элементам, относящимся к выявленному отклонению. По результатам анализа делается окончательный вывод о возможном наличии/отсутствии атаки (возможно, с указанием вероятности наличия атаки). Отметим, что реализация в режиме реального времени столь мощного программного аппарата требует наличия больших вычислительных мощностей, что является необходимым условиям для эффективной системы обнаружения скрытых вторжений.

Перечислим основные классы методов, которые могут быть использованы на этом в процессе анализа данных:

1. Статистический анализ. Основной задачей использования методов статистического анализа является выявление закономерностей нарушений типовых стабильных или стационарных показателей, даже незначительных, с целью построения базы знаний по эффективному поиску скрытых атак. Такая база должна содержать актуальные сведения об изменениях в пользовательских ресурсах (пароли, окружение), об изменениях в системных и пользовательских программах, файлах. Например, в перечне типовых сайтов 
одного из сотрудников появились новые ссылки, скачки в трафике и др. $[2$, c. 70$]$.

2. Аналитический метод. С помощью аналитических методов проводится общий анализ ситуации с общесистемных позиций с привлечением различных теоретических методов научного анализа (в частности, системного анализа, моделирования, исследования операций и др.). В результате выявляются все потенциально возможные источники атак. Затем проводится всесторонний анализ всего полученного перечня потенциальных атак, связанных с ними ситуаций и на этой основе, выявляются возможные новые сценарии реализации угроз. Далее текущая ситуация и сценарий ее развития сравниваются с полученным перечнем возможных сценариев и ситуаций, и выбираются одна или несколько наиболее близких по ситуации и сценарию развития атак [3, с. 30].

3. Эвристический метод. В основе практически любого эвристического метода лежит некое разумное, но недоказанное предположение, которое позволяет значимо, а часто существенно ускорить процедуру поиска. При этом метод реализуется («разворачивается») с условием, что эвристическое предположение истинное. Таким образом, эффективность конкретного эвристического метода в значительной степени зависит от того, насколько выбранное предположение близко к истинному положению дел. В системах противодействия атакам (вторжениям) точность сделанного предположения особенно актуальна, поскольку эти системы должны функционировать в режиме реального времени [4, с. 107].

4. Методы поиска слабо проявляемых связей и закономерностей в рядах данных. В частности, можно рассматривать методы Data Mining интеллектуального анализа данных. История возникновения этих методов связана с тем, что многие крупные торговые компании в течение десятилетий накопили огромные массивы данных, отражающих в себе различные особенности, а также различные аспекты поведении клиентов. Встала задача использования таких накопленных данных в интересах бизнеса, что привело к возникновению направления поиска Data Mining со своими методами анализа данных [5, с. 28]. К сожалению, ввиду коммерческой составляющей разработки и исследования в частных компаниях закрыты и недоступны широкой научной аудитории. Тем не менее есть публикации по этой тематике в открытой литературе. В системах защиты информации эти методы интересны, прежде всего, для поиска закономерностей (в частности, 
связанных с угрозами и атаками в сфере ИБ) по слабо выраженным их проявлениям. Как правило, результаты этих исследований засекречены. С точки зрения ИБ - выявление скрытых закономерностей осуществления угроз, зависимостей и т.д.

5. Метод нейронных сетей. Основное достоинство нейронных сетей по сравнению с перечисленными выше классами методов заключается в том, что: в нейронную сеть заложен механизм обучения (по образцу, по примерам самообучения). Чем дольше идет обучение, тем эффективнее себя ведет нейронная сеть. Практика показала (например, при решении задачи распознавание лиц), что данный метод при достаточно правильном и длительном обучении позволяет очень эффективно решать многие типовые задачи. Более того, существуют обоснованные прогнозы, что через два, три десятилетия в очень многих специальностях человек будет вытеснен системами искусственного интеллекта, опирающимся на нейронные сети. Применительно к сфере информационной безопасности, нейронные сети могут быть использованы и для систем, в которых условия функционирования изменяются (что типично для многих систем обеспечения ИБ), если ввести параметры, характеризующие эти условия, в качестве входных данных нейронной сети. Подобные сети могут быть адаптированы для распознавания новых механизмов вторжения, что важно применительно к поставленной в работе цели исследования [6, с. 7]. Отметим тем не менее, что использование одних только нейронных сетей в сфере обеспечения ИБ недостаточно ввиду непредсказуемого многообразия возможных способов совершения атаки на информационные активы объекта защиты.

6. Методы искусственного интеллекта (ИИ). Эти методы аккумулируют в себе эмпирический опыт и теоретические наработки экспертов и специалистов в сфере ИБ. Применительно к сфере обеспечения ИБ в рамках соответствующих систем ИИ создаются наборы сценариев, профилей и ситуаций, характеризующих различные варианты совершения атаки и вторжений. Реальные ситуации сравниваются с этими вариантами, и при возникновении большой близости реальной ситуации с одним из вариантов, приведенных в системе ИИ, эти обе ситуации могут быть отождествлены; затем реализованы соответствующие действия по нейтрализации атак, приведенные в системе ИИ. Основным элементом процесса обнаружения вторжения на основе методов ИИ является поиск аналогов, прецедентов (с точными или приближенным совпадением), что предполагает разработку процедур оценки близости ситуаций, профилей [7, с. 381]. 
Важное место в классе методов ИИ занимают методы продукционных правил, которые в своей основе также опираются на эмпирический опыт и теоретические наработки. Очень часто эти методы выделяют в отдельный самостоятельный класс. Основное достоинство этих методов - они позволяют охватить и включить в базу продукционных правил даже единичный опыт и индивидуальные представления отдельных экспертов. Применительно к сфере обеспечения ИБ серьезной проблемой может оказаться возникновение противоречий между отдельными правилами. Простое избавление от части противоречивых правил нежелательно, поскольку коллективный опыт в данной весьма закрытой сфере представляет большую ценность. Поэтому целесообразно найти способы совмещения подобных противоречивых правил на основе их уточнения с участием соответствующих экспертов.

Этап 3. Поиск и выбор средств противодействия новым угрозам и атакам, а также их реализация. Выявление наиболее эффективных средств нейтрализации потенциальных или реальных угроз является отдельной самостоятельной задачей, по своим методам слабо коррелированной с процессом мониторинга и выявления возможных атак. Таким образом, последовательность реализации методов нейтрализации угроз/атак и будет составлять содержание процедуры реализации процесса нейтрализации [8, с. 92].

Этап 4. Анализ результатов и подготовка отчетов. На этом этапе осуществляется анализ полученных результатов и подготовка отчетов. В отчётах необходимо фиксировать все полученные результаты знаний в целях последующего их использования: в нейронных сетях - при их обучении; в эвристических процедурах - с целью уточнения их параметров и значимости отдельных из этих методов; в системах ИИ, включая продукционные правила. Анализ результатов можно проводить как в соответствии с требованиями существующих стандартов, так и с помощью собственных разработанных средств и методов [9, с. 88].

Перечислим некоторые основные регламентные работы, которые должны быть охвачены в процессе мониторинга с целью выявления явных или скрытых вторжений:

1. Отчеты о безопасности пользовательских ресурсов. То есть служба ИБ должна контролировать пользовательские ресурсы (глубина контроля зависит от политики безопасности). В частности, контролироваться наличие пользователей без паролей, уязвимости пользовательского окружения, правильная установка каталогов и др. 
2. Проверка конфигурации программного обеспечения ИС. Не должно быть программ, не входящих в реестр разрешенных и/или установленных под контролем службы ИБ.

3. Выявление изменений в системных файлах - это элемент контроля целостности системы. Изменения могут производиться, но при этом они должны быть зафиксированы администратором.

4. Проверка прав доступа и других атрибутов системных файлов, команд, утилит, таблиц.

\section{Выводы}

Проведенный выше анализ позволяет сделать вывод, что наиболее привлекательными из перечисленных методов являются методы нейронных сетей и методы ИИ. Однако, методы ИИ достаточно сложны в реализации и сопровождении, что значительно затрудняет их практическое использование. В этой связи, наиболее привлекательными становятся методы нейронных сетей, которые имеют более простую идеологию реализации.

Подведем заключительные итоги выше сказанному. Разработанные этапы проведения мониторинга скрытых вторжений/атак позволяют напрямую выйти на процесс построения нейронной сети на основе совокупности всех возможных характеристик, способных оказать влияние на объект или систему защиты информации. В рамках последовательного проведения работ по гранту РФФИ №19-01-00383, разработка процедуры выявления вторжений на основе нейронной сети является следующей задачей, решение которой будет опубликовано в ближайшее время.

Благодарности. Работа выполнена при финансовой поддержке гранта РФФИ № 19-01-00383.

\section{Список литературы}

1. Попов Г.А., Симаворян С.Ж., Симонян А.Р., Улитина Е.И. Моделирование процесса мониторинга систем информационной безопасности на основе систем массового обслуживания // Информатика и ее применения. 2020. - T. 14. - № 1. - С. 71-79.

2. Кучер В.А., Путято М.М., Макарян А.С., Жданова Н.В. Применение математических методов анализа при формировании реестров инцидентов информационной безопасности для адаптивной системы защиты // Электронный сетевой политематический журнал "Научные труды КубГТУ". 2021. - № 1. - С. 66-78. 
3. Кошелев С.О., Ищенко К.И. Современные аналитические методы защиты информации корпоративных систем // Молодой ученый. - 2016. - № 28 (132). - C. 29-31.

4. Марков А.С., Матвеев В.А., Фадин А.А., Цирлов В.Л. Эвристический анализ безопасности программного кода // Вестник Московского государственного технического университета им. Н.Э. Баумана. Серия Приборостроение. - 2016. - № 1 (106). - С. 98-111.

5. Львович И.Я., Кравцова Н.Е., Чупринская Ю.Л Об использовании подходов, базирующихся на технологии DATA MINING // Вестник Воронежского института высоких технологий. - 2020. - № 1 (32). - С. 28-30.

6. Симаворян С.Ж., Симонян А.Р., Попов Г.А., Улитина Е.И. Процедура выявления вторжений в системах информационной безопасности на основе использования нейронных сетей // Программные системы и вычислительные методы. - 2020. - № 3. - С. 1-9.

7. Филяк П.Ю., Ермолин А.Н., Корецкий М.А. Об обеспечении информационной безопасности на основе использования искусственного интеллекта // Информация и безопасность. - 2020. - Т. 23. - № 3 (4). C. 379-388.

8. Альтерман А.Д., Лушников Н.Д., Гильмияров А.И. Методы нейтрализации хакерских атак // B сборнике: Перспективные научные исследования: опыт, проблемы и перспективы развития. Сборник статей по материалам международной научно-практической конференции. - Уфа: ООО "Научно-издательский центр "Вестник науки", 2019. - С. 91-94.

9. Агринский Н.М. Количественный анализ рисков информационной безопасности с необходимой точностью в соответствии требованиями международного стандарта ISO 27001:2013 // Инновации и инвестиции. - 2020. - № 6. - С. 88-92. 